\title{
Entre Poznan et Copenhague : le régime climatique au milieu du gué
}

\author{
Amy Dahan \\ Historienne et sociologue des sciences, UMR8560 Centre Alexandre Koyré, CNRS, 27 rue Damesme, 75013 Paris, France
}

\author{
Mots-clés : \\ $\mathrm{CdP}$; \\ gouvernance \\ climatique; \\ post-Kyoto ; \\ énergie ; \\ ONG
}

Keywords:
CoP;
climate governance;
post-Kyoto;
energy;
NGOs

\begin{abstract}
Résumé - Le but de ce texte est d'informer la communauté scientifique sur les conférences des Nations unies sur le changement climatique et ce qui s'y joue, en particulier à l'approche du terme de la première période d'engagement. Le texte situe la Conférence de Poznan dans l'histoire longue des négociations climatiques, en analysant en particulier l'évolution des clivages apparus, le rôle des pays en développement, celui du GIEC et des ONG. Il esquisse la culture du « off» de la Conférence pour donner une idée de la complexité de l'événement - qui ne se réduit pas à la négociation. Enfin, il résume les grandes options actuelles et leurs enjeux géopolitiques, et s'interroge sur le futur du processus international de gouvernance climatique, au-delà de l'échéance de Copenhague (décembre 2009).
\end{abstract}

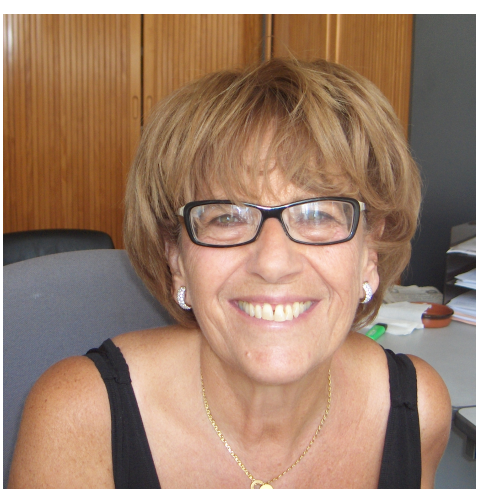

Auteur correspondant :

Amy.Dahan-Dalmedico@damesme.cnrs.fr

Directrice de recherche au CNRS, directrice adjointe du Centre Alexandre Koyré (CNRS-EHESS-MNHN), A. Dahan dirige l'équipe "Changement climatique, expertise et fabrication des futurs ». Elle est membre de l'Institut francilien Recherche Innovation Société (IFRIS) et du Réseau de recherche sur le développement soutenable (R2DS) de l'Île-de-France ( $c f$. http: //www.koyre.cnrs.fr/article.php3?id_article=200).

\section{Introduction}

La $14^{\mathrm{e}}$ Conférence des Nations unies sur le changement climatique, réunie à Poznan du $1^{\text {er }}$ au 12 décembre 2008, $\mathrm{s}^{\prime}$ est achevée dans une atmosphère tendue et morose. Les médias se sont plu à souligner qu'aucun progrès notable n'avait été accompli dans la préparation du futur traité, censé devoir être adopté à Copenhague en décembre 2009 pour prendre la suite du Protocole de Kyoto. Plus généralement, une partie importante des engagements pris dans le cadre de Kyoto n'ayant pas jusqu'ici été tenue, la crédibilité même du processus est l'objet de nombreuses interrogations, si ce n'est de scepticisme. Cependant, le processus de Kyoto reste pour beaucoup un symbole, une tentative de gouvernance mondiale face à un défi majeur pour l'humanité. Les Conférences, lieux des négociations entre les États parties prenantes, en sont un moment important. Elles sont aussi l'occasion périodique d'une mise en visibilité de l'alerte climatique, laquelle bénéficie incontestablement d'une prise de conscience croissante tant de la part des populations que des responsables politiques. 
Le but de ce texte ${ }^{1}$ est d'informer la communauté scientifique sur ce type de conférence et ce qui s'y joue, en particulier à l'approche du terme de la première période d'engagement. Pour cela : 1) le texte situera Poznan dans l'histoire longue des négociations climatiques; 2) une esquisse de la culture du « off » de la Conférence donnera une idée de la complexité de l'événement - qui ne se réduit pas à la négociation ; 3 ) chemin faisant, les grandes options actuelles et leurs enjeux géopolitiques seront abordés; 4) enfin, les derniers points porteront sur Copenhague et le futur du processus international de gouvernance climatique.

\section{La Conférence de Poznan dans le régime climatique}

\section{Un dispositif complexe}

La Convention-cadre des Nations unies sur les changements climatiques ${ }^{2}$ a été ouverte à la signature lors de la Conférence de Rio en 1992, avec la Convention pour la diversité biologique et la Convention contre la désertification. Comme ces dernières, elle est régie par deux principes onusiens : l'égalité de chaque pays, qui équivaut à une voix, et le principe - ô combien délicat à interpréter et à appliquer - de « responsabilité commune mais différenciée ${ }^{3} »$. Depuis 1995, se tient chaque année, dans la première quinzaine de décembre, une Conférence des Parties à la Convention (192 pays à ce jour). En 1997, le Protocole de Kyoto a été initié et proposé à la ratification, puis il est entré en vigueur en 2005, après que 175 pays - mais non les États-Unis - l'eurent ratifié. Depuis ce moment, lors des conférences climatiques, deux assemblées d'États se réunissent, formées l'une par les Parties à la Convention, l'autre par les Parties au Protocole de Kyoto ${ }^{4}$.

On peut simplifier la présentation des deux organes de gouvernance majeurs du dispositif en distinguant

\footnotetext{
${ }^{1}$ Ce texte est directement inspiré d'un rapport de recherche rédigé sous ma direction en février 2009, avec la collaboration de Stefan Aykut, Hélène Guillemot et Agatha Korczak, à l'issue d'une mission commune à Poznan. Cf. Dahan, A., Aykut, S., Guillemot, H., Korczak, A., 2009. Les Arènes climatiques : forums du futur ou foires aux palabres? La Conférence de Poznan. Rapport de recherche, Centre Alexandre Koyré, Paris (http://www.koyre. cnrs.fr/article.php3?id_article=697).

2 En français CCNUCC, en anglais UNFCCC.

${ }^{3}$ Principe inscrit à l'article 7 de la Convention de Rio.

${ }^{4}$ En anglais, Conference of Parties (CoP) et Meeting of Parties (MoP). À Poznan, se sont tenues la CoP 14 et la MoP 4.
}

deux fonctions ${ }^{5}$. À l'amont, le SBSTA ${ }^{6}$, organisme d'expertise, examine les questions scientifiques et techniques, assume l'expression politique des controverses apparues au sein des $\mathrm{CoP}$ sur ces dernières, fait le lien entre les scientifiques et les gouvernements ${ }^{7}$. En aval, le $\mathrm{SBI}^{8} \mathrm{~s}^{\prime} \mathrm{oc}-$ cupe de la mise en œuvre des décisions adoptées par la Convention et, notamment, des mécanismes de financement. À Poznan, s'ajoutaient des groupes spéciaux, censés accueillir les discussions, d'une part sur les bases d'un prochain accord post-2012 ${ }^{9}$, d'autre part sur les nouveaux engagements pour les grands pays émetteurs en $1990^{10}$, et divers groupes ou sous-groupes de travail que les coalitions d'États (pays de l'annexe $1^{11}, \mathrm{G} 77+$ Chine $^{12}$, pays les moins avancés, $\mathrm{AOSIS}^{13}$, etc.) ne manquent pas de convoquer. La multiplication de ces groupes entraîne une complexité et une difficulté à suivre les négociations, tant pour les pays que pour les observateurs.

Il existe aussi un comité d'observance ${ }^{14}$ censé inviter les pays à se mettre en conformité avec leurs engagements, dans des délais adéquats. Mais, étant donné que les Parties ont négocié volontairement et que la souveraineté des pays est inviolable, un État en défaut ne peut être sanctionné; il peut tout au plus être aidé. L'architecture de la gouvernance onusienne est conçue de manière à satisfaire toutes les demandes des Parties; sa complexité est donc inhérente à la diversité des intérêts et des positions des États membres. Un des enjeux actuels est justement d'arriver à trouver un compromis sur une architecture

\footnotetext{
${ }^{5}$ Cette architecture avec 2 organes est présente dans d'autres Conventions, notamment la Convention sur la diversité biologique.

6 Subsidiary Body for Scientific and Technological Advice (en français, Organe subsidiaire de conseil scientifique et technologique [OSAST]).

7 Sur les relations complexes entre le GIEC et le SBSTA dans les années 1990, cf. Miller, C.A., 2001. Challenges in the application of science to global affairs : Contingency, trust and moral order, in Miller, C.A., Edwards, P.N. (Eds), Changing the Atmosphere : Expert Knowledge and Environmental Governance, Cambridge (MA), The MIT Press, 247-285. Cf. aussi Dahan, A., 2008. Climate expertise : Between scientific credibility and geopolitical imperatives, Interdisciplinary Science Reviews, 33, 1, 71-81.

8 Subsidiary Body for Implementation (en français, Organe subsidiaire de mise en œuvre [OSMOE]).

9 AWG-LCA : Ad Hoc Working Group on Long-term Cooperative Action under the Convention (en français, GTS-ACV). Il relève de la $\mathrm{CoP}$.

10 AWG-KP : Ad Hoc Working Group on Further Commitments for Annex I Parties under Kyoto Protocol (en français, GTS-PK). Il relève des MoP.

11 Ils correspondent aux pays ayant les plus hauts taux d'émission de gaz à effet de serre (GES) en 1990.

12 Le groupe des 77, qui comprend aujourd'hui 130 pays, est souvent nommé «les pays en développement».

13 Alliance of Small Island States.

14 En anglais, Compliance Committee.
} 
simplifiée pouvant être adoptée après 2012. Quant au pouvoir exécutif, il est détenu par le Secrétariat des changements climatiques de la Convention - cheville ouvrière de cette énorme machinerie -, qui fixe l'ordre du jour; toutefois, il n'exprime pas de vision stratégique et a peu de moyens. Les organes se réunissent plusieurs fois par an pour avancer sur leurs mandats respectifs.

\section{Des questions et des clivages qui évoluent}

En quinze ans, la négociation climatique a beaucoup évolué et les grands clivages géopolitiques se sont largement déplacés. Pour l'essentiel, elle est passée d'une négociation entre les pays du Nord dans les années 1990 à une négociation Nord/Sud, ou encore des pays industrialisés avec les pays en émergence et en développement, aujourd'hui. Au moment de la discussion du Protocole de Kyoto (entre 1994 et 1997), l'enjeu politique principal était la répartition des objectifs de réduction des émissions de GES : les principaux protagonistes du débat étaient les États-Unis, l'Europe, le Japon et les pays dits en transition de l'ex-Union soviétique. Les pays en développement (PED) n'étaient pas concernés par ces objectifs. D'ailleurs, à cette époque, ils refusaient de considérer le changement climatique comme un problème global - c'est-à-dire posant la question de limites environnementales globales et le voyaient comme un problème de surconsommation du Nord. Au cours de la première phase des négociations, ils ont exprimé une insatisfaction croissante vis-à-vis du cadrage politique de la question climatique, trop marquée, selon eux, par le poids des sciences dures et le langage de la modélisation numérique. En effet, transférée dans le cadre politique, cette méthode efface le passé, naturalise le présent et globalise le futur ${ }^{15}$. Prendre un moment initial de référence - l'année 1990 - dans le Protocole leur semblait particulièrement inacceptable. Après avoir longtemps considéré que l'alerte climatique n'était qu'un cauchemar fabriqué pour contraindre et empêcher leur développement, les PED se sont lentement familiarisés avec le problème.

Plusieurs facteurs sont à l'origine de cette évolution : le rôle et l'action du Groupe intergouvernemental d'experts sur l'évolution du climat $\left(\right.$ GIEC $\left.^{16}\right)$ sont ici essentiels. L'attention particulière que le GIEC a accepté de porter aux pays du Sud, l'expertise réflexive qu'il a réussi à promouvoir, l'intégration de scientifiques issus du Sud dans l'élaboration et la rédaction des grands rapports, la manière dont il a négocié avec le SBSTA un partage des responsabilités (et une prise en main des questions

\footnotetext{
15 Cf. supra, note 1 (p. 22 du rapport), et Dahan Dalmedico, A., 2007. Le régime climatique entre science, expertise et politique, in Dahan Dalmedico, A. (Ed.), Les Modèles du futur. Changement climatique et scénarios économiques : enjeux scientifiques et politiques, Paris, La Découverte, p. 133 sqq.

16 En anglais, IPCC.
}

techniques ${ }^{17}$ ) sont autant d'éléments qui lui ont valu de conforter sa légitimité scientifique tout en gagnant le respect des politiques ${ }^{18}$. De plus, l'alliance singulière et durable qui s'est établie entre les scientifiques du GIEC et les ONG environnementales - à fronts renversés des rapports entre experts et représentants de la société civile dans d'autres situations de risques - a été cruciale.

Dans les années 2000, les pays en développement ont mis en avant le thème de $l^{\prime}$ " "adaptation » comme thème central dans les négociations. Cela témoigne d'un revirement de perspective : en bref, le changement climatique est déjà en cours, les PED sont les pays les plus vulnérables, il faut les aider à s'y adapter. Une convergence de fait s'est cristallisée un moment entre les Etats-Unis, qui voulaient retarder le temps de l'action et acceptaient d'évoquer les vulnérabilités des pays du Sud, et ces derniers, qui voyaient là une source de financements supplémentaires sans contraindre leur développement et sans aborder ce qui se passerait après 2012. Le GIEC a accepté avec enthousiasme de consacrer des recherches à l'adaptation et son groupe II, qui s'occupe des impacts et des vulnérabilités, a gagné en importance et en influence ; le quatrième rapport de 2007 a fait une très large place à ces questions. L'Europe s'est finalement ralliée à l'adaptation, tout en ne voulant pas la dissocier - contrairement aux États-Unis des réductions. À Poznan, les représentants des pays du Sud, en particulier les plus vulnérables aux événements extrêmes, non seulement ne contestaient plus le risque climatique d'origine humaine, mais ils lui ont imputé souvent dans leurs discours les catastrophes récemment subies - cyclones, sécheresses, inondations -, alors que les scientifiques sont traditionnellement plus prudents.

À la précédente Conférence de Bali, en décembre 2007, la négociation avait presque capoté dans les dernières heures de la Conférence au cours d'un débat politique très dur. D'un côté, les PED refusaient tous les engagements à 2050, ne voulant acter aucune contrainte à leur développement, tout en demandant en revanche des engagements à l'échéance de 2020 pour les pays industrialisés en vue de préparer la suite de Kyoto. De l'autre côté, les États-Unis refusaient les contraintes pour 2020 mais demandaient une contrainte pour 2050, reconnaissant enfin (!) le problème climatique comme sérieux. Quant à l'Europe, tiraillée entre les deux, elle acceptait les contraintes, tant pour 2020 que pour 2050. Les délégués ont finalement dû renoncer à toute mention d'horizon temporel et ils ont adopté une feuille de route qui a sauvé la CoP.

\footnotetext{
17 Il a su préparer des rapports sur les problèmes nombreux les concernant : usage des sols, questions agricoles ou forestières, guidelines pour l'évaluation nationale des émissions...

18 Cette position a été consacrée en décembre 2007 par l'attribution au GIEC du prix Nobel de la paix, conjointement à Al Gore. Sur le GIEC, cf. supra, note 15 (Dahan, 2007) et note 7 (Dahan, 2008).
} 
Ce qui a dès lors été appelé le «mandat de Bali» constitue un agenda autour de quatre éléments constitutifs dont tous les pays ont jugé qu'ils devaient être discutés désormais ensemble, d'ici à décembre 2009 (CoP de Copenhague), date à laquelle devrait être adopté un traité qui, après ratification de tous les États, prendrait la suite du Protocole de Kyoto. Ces éléments sont : les actions pour réduire les émissions de GES, les solutions d'adaptation aux impacts des changements climatiques, les transferts technologiques et les mécanismes financiers. Le débat sur la signification stratégique d'ensemble de ces quatre piliers (ce qu'on nomme la «vision commune») est resté à Bali en suspens. Néanmoins, sans répondre à la question épineuse de la nature juridique du futur accord, le mandat de Bali constituait une base assez cohérente pour le cycle de négociations en vue d'un régime post2012 et mettait les travaux à Poznan largement sur les rails.

L'importance des pays en développement est tout à fait frappante dans les CoP. Elle s'exprime par leur nombre (130 pays), les événements qu'ils organisent dans le off, la documentation qu'ils distribuent et leur activisme dans la négociation officielle. Cela ne concerne pas seulement les puissances émergentes, futurs gros émetteurs courtisés, mais aussi les pays les moins avancés (PMA), qui ont acquis dans cette enceinte un poids sans commune mesure avec leurs émissions. La Chine est, depuis 2007, le plus gros émetteur mondial (par an) de gaz à effet de serre et sa croissance industrielle et économique exceptionnelle en fait l'un des acteurs majeurs de la nouvelle géopolitique du carbone. Elle bénéficie aussi d'une aura incontestable au sein des pays du Sud; sa position est donc centrale à tous égards dans le processus des négociations. Mais, pays encore en développement, avec un PIB par habitant en dessous de la moyenne mondiale et une quantité d'émissions par habitant six fois moindre que celle des États-Unis, elle a toujours joué dans les CoP la carte des PED. Dans les négociations sur le climat, elle appartient au groupe dit G77 + Chine. Or, la solidarité des PED entre eux a été, depuis le début, leur stratégie immuable pour influencer les négociations. En dépit des différences économiques massives et des intérêts disparates, ils ont tenté de construire des positions communes au sein du groupe en insistant sur la responsabilité historique du monde industrialisé et sur la disparité des montants d'émissions per capita. L'alliance de la Chine avec le G77 n'a jamais failli de ce point de vue.

Avec l'ascension du thème de l'adaptation, les PED ont profondément reconfiguré la négociation climatique et introduit leurs préoccupations. La signification de cette victoire, confirmée à Poznan, est stratégique : en l'absence - depuis Johannesburg, en 2002 - d'autres forums internationaux de grande envergure consacrés au développement, le changement climatique est devenu la driving force de tout le champ du développement. Cela signifie que toutes les autres questions environnementales (biodiversité, eau...), de développement ou d'équité NordSud, qui composaient, depuis les années 1970, l'ensemble des questions concernées par le développement durable, sont désormais dans l'orbite du régime climatique, soumises au rythme de sa progression et de la dynamique géopolitique qui s'y déploie.

Deux éléments importants du contexte immédiat de Poznan ont pesé très lourd sur le déroulement de la CoP. Premièrement, l'élection de Barack Obama, chargée d'espoirs, laissait un vide transitoire, renforçant l'idée que les grandes avancées ne pourraient intervenir à cette échéance. Deuxièmement, le fait que la Conférence ait lieu en Europe, sous présidence française.

Depuis le retrait des États-Unis des négociations, l'Europe a joué un rôle moteur dans le processus, en adoptant, de façon unilatérale à la veille des dates-clés du processus, des objectifs de réduction des émissions de GES. Elle est le seul groupe d'États à avoir signé le protocole de Kyoto et le climat représente, pour ses instances, l'un des (rares) succès internationaux. À Poznan, on attendait qu'elle donne un nouvel élan aux négociations ${ }^{19}$, $\mathrm{d}^{\prime}$ autant que les résultats concrets des politiques climatiques européennes sont jugés décevants ${ }^{20}$. Pour répondre à ces critiques et reprendre l'initiative, l'Union européenne a discuté en interne l'adoption d'un «paquet énergie-climat ${ }^{21}$ » avec un triple objectif affiché pour 2020 : réduction d'au moins $20 \%$ des émissions de GES par rapport à 1990 (objectif pouvant atteindre $30 \%$ dans le cas d'un accord satisfaisant à Copenhague, c'est-à-dire d'un effort comparable des autres pays développés); $20 \%$ d'énergies renouvelables dans le bouquet énergétique européen ; enfin, réduction de la consommation énergétique de $20 \%$ par rapport au niveau prévu pour 2020 par une amélioration de l'efficacité énergétique ${ }^{22}$. Or, ce « 3 fois 20 » a peiné à convaincre : les débats à Bruxelles se sont déroulés en même temps que la Conférence de Poznan, exposant au grand jour toutes les hésitations et les concessions à certains pays et

\footnotetext{
${ }^{19}$ Cf. Radanne, P., 2008. Les leçons de Bali, L'Encyclopédie du développement durable, 56 (http://encyclopedie-dd.org/ La-lecon-de-Bali).

${ }^{20}$ L'Union à quinze (celle qui a signé Kyoto) a réduit globalement ses émissions de GES de 2,7\% entre 1990 et 2006, et reste bien loin de son objectif de $8 \%$ pour 2008-2012 (Cf. European Environment Agency, 2008. Annual European Community Greenhouse Gas Inventory 1990-2006 and Inventory Report 2008 : Submission to the UNFCCC Secretariat, EEA Technical Report, 6/2008, p. 11).

${ }^{21}$ Le paquet regroupe plusieurs textes, adoptés par le Parlement européen. Une synthèse des discussions se trouve en ligne (http://ec.europa.eu/environment/climat/climate_action.htm).

22 Un autre objectif, plus controversé mais moins médiatisé, est d'atteindre une proportion de $10 \%$ de biocarburants dans la consommation totale des véhicules d'ici 2020.
} 
à certaines industries ${ }^{23}$. Bien qu'un accord entre les 27 ait été trouvé in extremis à Bruxelles, au dernier jour de la Conférence de Poznan, les difficultés pour l'obtenir, le fait qu'il soit en partie vidé de sa substance, ainsi que l'absence de proposition concrète sur la question du financement de l'adaptation des PED n'ont pas permis à l'UE de se placer en position de force dans les négociations.

\section{La culture du off de la Conférence}

Une CoP ne se réduit pas aux négociations : à côté du in, il y a un énorme off auquel des milliers de personnes participent ${ }^{24}$. Plusieurs cercles d'événements parallèles s'y déroulent simultanément : ateliers de 2 ou 3 heures, rencontres d'une journée ou deux, colloques satellites, expositions, etc. Ces événements sont organisés par un État, une institution académique, un think tank, une agence internationale, des organisations sociales ou syndicales, des groupements d'entreprise, des chambres de commerce, des ONG, petites ou grandes, locales ou globales, se consacrant à la protection de la nature, à l'environnement ou au développement de pays du Sud ${ }^{25}$. Le plus souvent, deux ou trois partenaires proches s'associent pour organiser des événements et les associations ne sont jamais fortuites. Par exemple, la Chine organise son side event avec le Programme des Nations unies pour le développement (PNUD), affirmant haut et fort son attachement au cadre onusien, tandis que le PNUD la félicite pour ses efforts de politique climatique nationale.

\section{Une multitude d'événements de statuts différents}

Adaptation, réductions, technologies et finances, les quatre piliers de la feuille de route de Bali ont structuré une grande partie des discussions de Poznan, dans le off comme dans le in. Les événements parallèles entretiennent, en effet, des rapports de différents types avec la négociation. Certains développent le point de vue d'un pays ou d'une organisation. L'Europe, la Chine, le Brésil, l'Inde en ont organisé dans cet esprit. Les négociateurs y participent parfois ou y envoient des assistants pour connaître les positions exprimées et prolonger les débats entamés dans des séances officielles, par exemple sur les transferts technologiques ou les questions financières. Les instituts de recherche, les think tanks ou les

\footnotetext{
${ }^{23}$ Cf. Third World Network, 2008. Blow to EU climate policy as big concessions made to industry, TWN Poznan News Update, 22, 1 (http://www.twnside.org.sg/climate.htm).

2410000 personnes à Poznan; 14000 à Bali, en 2007; 7000 à Nairobi, en 2006. Le public d'une CoP dépend du lieu où elle se réunit.

${ }^{25}$ On y distingue les INGOs (International Non-Governmental Organizations), les BINGOs (Business-oriented INGOs), les RINGOs (Research INGOs).
}

ONG présentent des travaux sur des thèmes proches de ceux débattus dans les négociations : modélisations économiques, scénarios, trajectoires et calendriers de réduction... Ils proposent les mesures associées, notamment le bouquet énergétique pour y parvenir, et servent d'appui à certains pays. Greenpeace a ainsi présenté son scénario énergétique global : «energy [r]evolution ${ }^{26}$ »; il est censé permettre une stabilisation de la concentration du $\mathrm{CO}_{2}$ dans l'atmosphère en dessous de 450 ppm en 2050 et garder ainsi le réchauffement climatique sous la barre jugée dangereuse (par le GIEC) de $2{ }^{\circ} \mathrm{C}$, sans développer le nucléaire et sans utiliser le piégeage et le stockage $\mathrm{du}$ dioxyde de carbone ${ }^{27}$. Cette étude très complète de Greenpeace s'inscrit dans un souci de rouvrir le débat énergétique ; elle veut montrer qu'il est possible de combattre le changement climatique en misant uniquement sur la maîtrise de l'énergie et les énergies renouvelables, en agissant sur la demande énergétique, et sans devoir recourir à des technologies jugées dangereuses ou polluantes. Le World Resources Institute (WRI) ${ }^{28}$ a exposé, quant à lui, un point de vue nettement favorable sur la séquestration et la capture du carbone, technologie qu'il qualifie de "pont vers un futur énergétique soutenable ${ }^{29}$ ", et il a présenté des analyses sectorielles. Autre exemple : l'Institute of Development Studies de l'Université du Sussex a proposé une vision politique « hybride» du futur régime climatique qui puisse être acceptée par le Nord et les pays en développement ${ }^{30}$. De telles initiatives ont un rôle important d'ouverture du champ des possibles. Les side events remplissent ainsi de multiples fonctions relativement aux négociations : vitrines de politiques climatiques des États, plateformes pour de nouvelles propositions, lieux de promotion de stratégies énergétiques ou industrielles (nucléaire, éolien, solaire, technologies du «charbon propre», efficacité énergétique).

Il y a aussi les colloques qui se tiennent dans les grands hôtels proches de la Convention. Deuxième cercle du off, témoignant de moyens importants, ces événements permettent à des acteurs d'affirmer un pouvoir politique, de

\footnotetext{
${ }^{26}$ Cf. Greenpeace International, European Renewable Energy Council, 2008. Energy [r]evolution: A sustainable global energy outlook, http://www.energyblueprint.info.

27 Cf. supra, note 15 : Dahan, 2007, loc. cit.

28 Le WRI, puissant centre de recherche américain, proche des démocrates, se définit comme "an environmental think tank that goes beyond research to find practical ways to protect the earth and improve people's lives » (http://www.wri.org/about). 29 Ibid.

${ }^{30}$ Cf. notamment Yamin, F., Depledge, J., 2004. The International Climate Change Regime: A Guide to Rules, Institutions and Procedures, Cambridge (UK), Cambridge University Press. Cette initiative préconisait notamment la création d'une mission permanente - un peu comme le Bureau international du travail se substituant aux conférences annuelles, jugées lourdes et peu efficaces.
} 
rendre visibles des réseaux d'influence et de populariser des positions. En voici quatre exemples.

Les Development and Climate Days se réunissent pendant le week-end au milieu de la Conférence ${ }^{31}$. Lancée en 2002 sous le titre de Adaptation Day, cette initiative a gagné en puissance au cours des années. Plusieurs institutions académiques $^{32}$ y sont impliquées et cette rencontre se veut clairement au service de la coalition des PMA et de la promotion, au sein de la Convention, de l'articulation étroite entre adaptation et développement. $Y$ ont pris la parole des membres d'ONG actifs dans ces pays, des chercheurs qui travaillent avec eux, des délégués, des financiers aussi, car l'un des sujets principaux concerne le financement de l'adaptation et les transferts vers les pays les plus démunis. La critique des positions de pays du Nord dans les négociations y est fréquente, mais l'atmosphère des discussions est généralement pragmatique et concrète.

Le Business Day est organisé par la Chambre internationale de commerce et le World Business Council for Sustainable Development, avec des responsables de haut niveau du monde des affaires, de la Banque mondiale, de grands groupes industriels, de l'ONU, des responsables politiques de ministères de l'Économie de divers pays (Japon, Europe, Afrique du Sud, etc.). Ici, on parle marché du carbone, efficacité énergétique, nouvelles technologies...

Le Forest Day 2 (qui a pris la suite de celui de Bali, qui fut un grand succès) a constitué à lui seul une miniconvention avec des plénières, des side events (une quarantaine), et près de 900 participants recouvrant tous les protagonistes dans ce domaine : experts, chercheurs académiques (forestiers, écologues...), représentants des peuples autochtones, décideurs de différentes parties du monde... Le résumé des délibérations, unanimement considérées comme fructueuses, fut transmis au secrétaire de la Convention, qui parrainait la rencontre.

Le colloque, plus institutionnel, organisé par l'Agence internationale de l'énergie (AIE) autour de ses modèles, scénarios et résultats, a marqué les esprits ${ }^{33}$. Le scénario de référence mondial (sans mise en œuvre de nouvelles politiques climatiques) qui y a été présenté ouvre en effet sur des perspectives et des chiffres vertigineux ; il est qualifié de suicidaire par 1'Agence. La demande énergétique va croître de 1,6 \% par an en moyenne entre 2006 et 2030,

\footnotetext{
31 Pour un compte rendu de cet événement, $c f$. http://www.iisd. $\mathrm{ca} /$ climate/cop14/dcd.

32 On retrouve notamment l'International Institute of Sustainable Development (IISD, Canada), auquel participe Saleem Huq, l'International Institute of Environment and Development (IIED, Royaume-Uni) et le Stockholm Environment Institute (SEI, Suède). Ces trois centres de recherche sont particulièrement actifs. Ils font, écrivent-ils dans leur documentation, de la science pour le politique.

${ }_{33}$ Cf. International Energy Agency, Organisation for Economic Co-operation and Development, 2008. World Energy Outlook, IEA, OECD (http://www.worldenergyoutlook.org/2008.asp).
}

et les sources fossiles y répondront pour $80 \%$. La Chine, l'Inde et le Moyen-Orient seront pour les trois quarts à l'origine de l'augmentation des émissions, qui correspondra à une hausse de la température moyenne de $6{ }^{\circ} \mathrm{C}$ ! En dépit de ses efforts pour intégrer prospective énergétique et lutte contre l'effet de serre, l'AIE, traditionnellement plus impliquée dans la sécurité énergétique que dans les questions écologiques, a été critiquée par divers acteurs. Les ONG lui reprochent de partir de chiffres trop élevés de demande énergétique et de miser sur une extension de l'offre (investissement dans l'exploitation des champs pétroliers, dans l'infrastructure énergétique et les raffineries, etc.), au lieu de promouvoir des politiques de maîtrise de l'énergie et des changements de comportement. Cette situation a inspiré au Climate Action Network (CAN), réseau regroupant plus de $430 \mathrm{ONG}$, un billet percutant sur les « deux cerveaux de $1^{\prime} \mathrm{AIE}^{34}$ ».

Les résultats et les chiffres-clés - extraits du $4^{\mathrm{e}}$ rapport du GIEC (qui servent de référence dans les CoP et les négociations) - indiquent que, pour atteindre l'objectif de stabilisation des concentrations de $\mathrm{CO}_{2}$ dans l'atmosphère à 450 ppm à l'horizon 2050, il faut que les émissions de GES soient au moins divisées par 2 en 2050, après avoir atteint un pic d'ici 10 à 15 ans et après une réduction en 2020 de 25 à $40 \%$ par rapport à 1990 pour les pays industrialisés ${ }^{35}$. Aucune comparaison précise - du point de vue de la répartition des efforts, des trajectoires de stabilisation, des résultats escomptés selon les divers secteurs - entre les scénarios de stabilisation de l'AIE (à 450 ppm et à 550 ppm) et celui de Greenpeace (à 450 ppm) n'a eu lieu dans aucune enceinte à Poznan; or, elle serait d'un immense intérêt.

\section{Le tunnel de l'énergie}

Le thème de l'énergie a connu cette année une ascension spectaculaire dans les travaux de la CoP. Bouquets énergétiques, scénarios d'évolution énergétique et technologies énergétiques ont massivement occupé les esprits et les débats dans le off. Par ce biais, derrière le changement climatique, se profile un nouvel ordre géopolitique où se conjuguent étroitement les préoccupations de sécurité énergétique et d'émissions de carbone. La prédominance de la question de l'énergie, associée à l'indicateur carbone, contribue à faire émerger un « effet tunnel » : toutes les activités économiques, agricoles et d'usages des sols ne sont plus jaugées qu'à cette aune-là. Le « tunnel de l'énergie » renforce le privilège énorme accordé à la technologie dans l'éventail des réponses au défi climatique. Et parmi les technologies, celle de la capture et de la

\footnotetext{
${ }^{34}$ Cf. Climate Action Network, 2008. The two brains of the IEA, ECO, CXVI, 7, 2 (http://www.climatenetwork.org/eco [Poznan ECOs; Poznan ECO 7]).

${ }^{35}$ C'est notamment l'objectif de l'Union européenne (cf. supra, note 1 : Dahan et al., 2009, p. 10 sq.).
} 
séquestration du carbone (CSC) est appelée à devenir le centre d'importantes controverses.

Paradoxalement, le tunnel de l'énergie s'accompagne aussi d'une absence relative de discussions sur les modes de consommation de l'énergie, c'est-à-dire sur les modes de vie en tant que tels (transports, habitat, urbanisation), sur les modes de production, de consommation de biens et de développement à venir. Est-ce par respect de la souveraineté des États? Toujours est-il que rien ne se dit jamais, par exemple, sur la destruction du développement rural des pays du Sud (pourtant dénoncée par de nombreux économistes du développement) ou sur l'urbanisation galopante, génératrice de pauvreté ${ }^{36}$. Rien non plus sur la dynamique de progression des technosciences et ses impasses. Plus généralement, peu de discours de contestation radicale, d'utopies ou simplement de paroles décalées (venus des ONG ou autres ${ }^{37}$ ) ont vu le jour. Paradoxalement encore, le tunnel de l'énergie a tendance à effacer la dimension proprement environnementale du changement climatique, au profit d'aspects principalement techniques.

\section{ONG et fabrique de l'opinion}

Plusieurs petits journaux édités quotidiennement par divers réseaux d'ONG rendent compte de tout ce qui se passe et se dit dans les principaux lieux de négociation ${ }^{38}$. Ces journaux sélectionnent aussi, dans l'énorme masse d'événements parallèles, ceux qu'ils souhaitent mettre en avant et dont ils résument les contenus principaux. Les ONG contribuent ainsi à la fabrique de l'opinion publique dans la CoP, elles attribuent chaque jour des Fossile Medals aux pays ou aux acteurs les plus récalcitrants dans les négociations. En retour, les décideurs peuvent prendre la température des questions cruciales et les ONG se font le relais entre des mobilisations (inter)nationales et la Convention. Les ONG et leurs bulletins quotidiens apparaissent comme des instruments de traduction entre la société civile et les décideurs politiques. Par exemple, l'accord à Bali sur la feuille de route n'a pu être finalement

\footnotetext{
36 Cf. Sachs, I., 2008. La Troisième Rive : à la recherche de l'écodéveloppement, Paris, Bourin. Cf. aussi sa bibliographie sur les villes, sur le Brésil, etc.

37 Le seul discours de rupture a été tenu le 7 décembre, hors de la CoP, dans une réunion de représentants de groupes (souvent proches d'Attac) pour organiser un rassemblement (baptisé Climate Justice) avant Copenhague. Le thème principal est celui de la dénonciation d'un capitalisme vert qui sera forcément autoritaire et justifiera, par la menace de la crise écologique, son ignorance des questions sociales ( $c f$. « 20 theses against green capitalism », http://klimacamp08.wordpress.com/2008/12/).

38 Il s'agit du Bulletin des négociations de la Terre, publié par l'IISD, de ECO, publié par le CAN, et de TWN, publié par le Third World Network (ONG basée à Penang en Malaisie, qui existe depuis 1984 et qui a été créée dans le but de « coordonner et consolider la coopération des ONG du développement dans le Sud » (http://www.twnside.org.sg/twnintro.htm).
}

obtenu qu'à la faveur d'un rapport de force auquel le off de la CoP a contribué de façon décisive.

On pourrait croire que tous les participants de la CoP partagent la seule conviction d'une action nécessaire, d'une urgence climatique. En fait, cohabitent des préoccupations, des motivations et des intérêts infiniment variés : risque climatique, oui, mais aussi développement des pays pauvres, priorités environnementales, opportunité d'un nouvel ordre mondial, place de tel groupe de pays, rôle de telle ONG, avenir de telle technologie... La lutte contre le changement climatique s'accommode de multiples visions du monde. Pourtant, malgré la diversité des publics et des intérêts, on voit peu de débats passionnés ou de déclarations hostiles. Il n'est jamais question non plus de remettre en cause ouvertement le cadre même des négociations. Les points de vue grandement divergents coexistent et se côtoient sans s'affronter directement. Les événements parallèles rassemblent, en effet, des assistances assez homogènes (diplomates et politiques ici, scientifiques et experts ailleurs, militants d'ONG, représentants de peuples autochtones, etc.) et les débats succédant aux interventions ont parfois semblé assez cadrés. Dans certains side events, des critiques s'expriment envers la politique des pays industrialisés ou envers les mécanismes de développement propre (MDP), l'un des trois mécanismes du Protocole de Kyoto, notamment lors d'ateliers portant sur la réduction de la déforestation (nouveau mécanisme Reduced Emissions from Deforestation and Degradation $\left[\right.$ REDD ${ }^{39}$ ). Mais ces critiques, partagées par les participants à la réunion, y restent confinées. Au-delà des différences, il existe un accord réel sur les « règles du jeu » de cette conférence et la civilité onusienne s'étend aux ateliers.

Une hiérarchie invisible traverse pourtant les différents groupes de participants à la $\mathrm{CoP}$, celle qui sépare le tout-venant du cercle plus étroit des experts... des CoP. Qu'ils soient négociateurs, délégués ou responsables d'ONG, ces habitués des conférences climatiques, d'appartenances et d'intérêts divers, ont en commun de mâ̂triser les textes juridiques foisonnants, l'organisation complexe des innombrables sous-groupes de négociations, ainsi que les références et le jargon onusiens, ce qui leur permet de discerner les influences et les enjeux essentiels dans la jungle toujours plus épaisse des négociations ${ }^{40}$. Ce noyau d'initiés des CoP semble constituer ce

\footnotetext{
${ }^{39}$ Cf. Climate Action Network, 2008. Setting REDD up for failure?, ECO, CXVI, 9, 2 (http://www.climatenetwork.org/eco [Poznan ECOs; Poznan ECO 9]).

${ }^{40}$ Le constat d'une complexité technique croissante est largement partagé, jusque dans les rangs des négociateurs euxmêmes : «Les négociateurs n'y voient plus très clair non plus $\mathrm{du}$ fait de textes juridiques extrêmement complexes, et en expansion permanente, qui se stratifient de réunion en réunion. La complexité de la forme rend le fond illisible et la prospective brouillée » (Radanne, P., 2008, Les leçons de Bali, loc. cit.).
} 
qu'un ancien habitué a appelé une «méta-communauté épistémique ».

Les organisations non gouvernementales environnementales ont joué dès le début un rôle majeur dans la mise en place du régime climatique, conférant à l'expertise du GIEC un crédit moral qui s'est avéré très précieux. Ces quelques années, elles ont été rejointes massivement par des organisations de recherche et des think tanks phénomène frappant qui illustre les relations nouvelles, très poreuses, entre monde académique et monde politique $^{41}$. Toutes ces ONG oscillent entre quatre fonctions : celle d'une société civile souhaitant exercer des pressions, celle de traducteurs et de relais entre divers mondes, celle - de plus en plus importante - de conseil et de soutien logistique pour les PED et, enfin, celle d'experts et de laboratoires d'idées ouvrant le champ des possibles $^{42}$. À Poznan, Greenpeace a rempli le mieux cette dernière fonction, mais le risque est perceptible de voir les ONG perdre leur capacité critique des paradigmes dominants.

En résumé de cet aperçu, on peut dire que, par les idées (scénarios, expériences, projets) qui y sont brassées, le off se présente comme le poumon de la négociation, un poumon qui alimente le processus du in et offre en retour un public, une caisse de résonance, un terrain d'apprentissage et d'exploration. Les conférences climatiques - et celle de Poznan n'est pas une exception - ne sont pas seulement des lieux de palabres, mais bien des « forums hybrides ${ }^{43}$ » réunissant un très grand nombre d'acteurs (politiques, industriels, scientifiques, citoyens, membres des ONG, activistes, etc.) qui se confrontent ensemble aux difficiles problèmes posés par l'effet de serre anthropique, en y recherchant des solutions concrètes et viables. L'approche pragmatique, qui caractérise cette quête, s'efforce de contourner les affrontements idéologiques, d'unifier les volontés; elle confère un caractère touffu et concret à l'ensemble des sujets abordés. Toutefois, alors que des conflits majeurs d'intérêts politiques sont présents, la lecture qui en prévaut dans le off est dominée par des agencements de dispositifs techniques et semble parfois euphémique.

${ }^{41}$ Le monde français de la recherche est nettement moins présent dans ces arènes que celui des pays anglo-saxons (Angleterre, Canada), d'Europe du Nord ou même d'Allemagne. Il semble plus réticent à occuper des fonctions assez nettement politiques.

${ }^{42}$ Cf. Dahan, A., Buffet, C., 2009. Environmental NGOs in the Raising Debate of Climate Change, in Marty, P., Devaux, S. (Eds), Social Movements and Public Action : Lessons from Environmental Issues, Prague, Publications du CEFRES.

${ }^{43}$ Cf. Callon, M., Lascoumes, P., Barthes, Y., 2001. Agir dans un monde incertain, Paris, Le Seuil (chapitre 1, «Forums hybrides », pp. 29-60). Nous reprenons sciemment la terminologie de ces auteurs bien qu'il existe des différences importantes entre les $\mathrm{CoP}$ et les exemples qu'ils traitent.

\section{Quel futur pour le processus?}

La question générale de l'avenir du processus est ouverte. La réponse au défi climatique peut-elle et doitelle continuer à se construire collectivement à travers lui, dans le cadre d'un régime climatique unique, sous l'égide des Nations unies? Un autre régime pourraitil être plus efficace? Les questions de l'adaptation, des transferts de technologies et financiers, ainsi que des PED semblent ne pas relever du même régime que les réductions d'émissions. Mais qui pourrait aujourd'hui définir une politique climatique efficace et équitable à l'échelle mondiale?

\section{Le cadre de l'ONU?}

L'ONU a eu un rôle historique majeur dans la constitution du régime climatique ${ }^{44}$ : elle a suscité, en 1988, la création du GIEC, devenu le fer de lance de ce régime; elle est aussi à l'origine de la Convention climat UNFCCC (Rio, 1992). Au moment des tensions politiques avec les pays en développement qui contestaient le cadrage du problème, elle a su modifier les structures de gouvernance de la Convention en créant le SBSTA et désamorcer un rejet possible des pays du Sud vis-à-vis du péril climatique. Aujourd'hui, dans la perspective d'une future architecture qui prendrait la suite du Protocole de Kyoto, l'ONU n'entend pas être dessaisie du dossier. Sa présence massive dans le off portait principalement sur deux des quatre piliers de la feuille de route de Bali - la finance (avec le poids de la Banque mondiale et du Fonds pour l'environnement mondial) et la technologie - ainsi que sur le renforcement des capacités d'action dans les pays en développement. À plusieurs reprises, l'ONU a voulu affirmer un partenariat stratégique avec la Chine, pour équilibrer sans doute les offres que les États-Unis ou le Japon lui faisaient d'être mieux prise en considération, notamment en accédant à des architectures hors de son orbite.

La discussion sur le mécanisme financier REDD, permettant de rémunérer les pays qui réduisent les émissions issues de la déforestation, a montré que, si le Secrétariat de la Convention sait prendre en compte les demandes des États, les compétences propres aux organisations onusiennes déjà existantes (biodiversité, changement climatique) conduisent à créer toujours plus de nouveaux circuits complexes de gouvernance. Ainsi, l'approche globale dans le cas des forêts n'a pu aboutir à

\footnotetext{
${ }^{44}$ Par l'intermédiaire de l'Organisation météorologique mondiale (OMM) et du Programme des Nations unies pour l'environnement (PNUE), deux organismes de l'ONU.
} 
la création d'un seul organe de gestion « holiste » de cette question $^{45}$.

L'ONU s'affirme comme le seul service public international capable de répondre de manière transversale à la question du changement climatique et d'aider les pays (pauvres) à y faire face. Mais mobiliser un système, par nature morcelé, ne fait qu'entraîner une multiplication des champs de compétences. De plus, un argument presque exclusivement orienté vers les pays en développement ne peut suffire à convaincre les grands États émetteurs du Nord, comme les États-Unis, de rester dans son giron. D’autres facteurs géopolitiques sont appelés inéluctablement à intervenir.

\section{Discuter une nouvelle architecture post-Kyoto?}

En fait, ceux qui critiquent violemment, voire rejettent le cadre et les formes de gouvernance du Protocole de Kyoto, ne s'expriment pas dans les enceintes des CoP. Quand on est dans cette arène politique, que ce soit le in ou le off, on se situe de facto dans le processus, quitte à vouloir infléchir des régulations, changer les rapports de force ou obtenir des compensations. Aucun acteur présent à Poznan n'a préconisé un autre cadre de gouvernance ou élaboré une structuration alternative à un traité international sous les auspices des Nations unies. Le gouvernement républicain de Georges W. Bush, qui avait refusé de ratifier le Protocole de Kyoto, le critiquait vivement, ainsi que la gouvernance onusienne. Mais les États-Unis ont fait volte-face dès 2007 en affirmant que la Convention était le cadre approprié pour les négociations pour un régime post-2012 ${ }^{46}$ et l'administration Obama a manifesté des signes clairs d'engagement pour des réductions domestiques et dans la négociation climatique internationale.

Une initiative mérite qu'on s'y arrête plus longuement et qu'on en élucide la fonction. Il s'agit du projet de l'Université Harvard sur l'International Climate Agreements piloté par Joseph E. Aldy et Robert N. Stavins ${ }^{47}$. En 2006,

45 Le REDD est rattaché au SBSTA dans le système UNFCCC, mais il fait aussi l'objet d'un programme intitulé UN-REDD qui regroupe la FAO, le PNUD et le PNUE, dédié aux pays en voie de développement. Ce programme a été lancé officiellement en septembre 2008 par Ban Ki-moon et le Premier ministre norvégien Jens Stoltenberg. L'initiative est soutenue par l'IUCN.

46 Ils ont fait ces déclarations dans le cadre du G8 (Heiligendamm, juin 2007) et de la réunion des dirigeants de l'Asia-Pacific Economic Cooperation [APEC] (Sydney, septembre 2007).

47 Cf. Aldy, J., Stavins, R.N., 2008. Designing the Post-Kyoto Climate Regime : Lessons from the Harvard Project on International Climate Agreements. Report, Harvard Project on International Climate Agreements, Belfer Center for Science and International Affairs, Harvard Kennedy School (http://belfercenter.ksg. harvard.edu/publication [Academic Papers \& Reports ; Reports ; November 24, 2008]). Stavins est « Albert Pratt Professor of Business and Government » (John F. Kennedy School of Government, à une époque encore éloignée de l'élection présidentielle américaine, un groupe de chercheurs économistes de cette université a décidé de lancer une consultation internationale et interdisciplinaire auprès de centres académiques, d'industries, de gouvernements et d'ONG pour « aider à identifier les principes d'une politique climatique postKyoto qui soit scientifiquement adéquate, économiquement rationnelle et politiquement pragmatique ».

Le rapport de Harvard s'ouvre sur les principes suivants : que l'on considère Kyoto comme un mauvais ou un bon premier pas, il faudra un nouvel accord pratique et réaliste, qui fasse une grande place au transfert et à l'innovation technologiques, et donne des motivations pour suivre les engagements pris et participer largement à l'effort général. L'accord devra être économiquement viable et cohérent avec les principes du commerce international. Il est clair que, dans la grammaire de la CoP, cela signifie qu'il devra fonctionner suivant les lois du marché et les principes du cap and trade (marché des permis d'émissions). Il devra combiner des objectifs à court et à long terme. Le problème du changement climatique étant global, la coopération des États est indispensable, qu'elle passe par la Convention, le G20 ou des accords bilatéraux. Les auteurs disent avoir identifié, au terme de leur consultation $^{48}$, quatre architectures potentielles. Il s'agit d'approches économiques stylisées (au sens d'idéaux types) qui pourraient se combiner et dont l'ensemble définit l'espace des possibles pour le futur des négociations. Précisons ces approches.

La première est construite à partir d'objectifs ciblés de réductions des émissions de GES et d'un calendrier de leur mise en œuvre, avec des plafonds d'émissions évoluant dans le temps, à partir d'une formule qui reflète les responsabilités différenciées des pays et qui tienne compte des réalités politiques présentes et des considérations d'équité à long terme. Les pays en développement ne seraient pas astreints au début, mais la situation devrait évoluer avec un facteur d'égalisation progressive. Outre les difficultés immenses d'un accord général de tous les pays sur les objectifs plafonds d'émissions et les calendriers de chacun d'entre eux, cette architecture politiquement ambitieuse implique aussi de réguler internationalement un système global de marché des émissions qui relierait les systèmes nationaux et régionaux (États de l'Union européenne ou des États-Unis).

La deuxième approche est fondée sur des politiques nationales, allant de pair avec un ensemble d'accords internationaux qui traiteraient séparément des différents secteurs et des différentes sources d'émissions, aussi bien que de questions cruciales pour le changement climatique,

Harvard University); Aldy est aujourd'hui dans l'équipe de Barack Obama.

48 À partir des retours de 28 groupes répartis dans le monde (Europe, États-Unis, Chine, Inde, Japon et Australie) qui y ont participé. 
comme la recherche et le développement des technologies, la déforestation et l'adaptation. Les accords sectoriels établiraient des standards globaux pour des industries ou des technologies spécifiques (par exemple, le CSC) ou des catégories de sources de gaz à effet de serre. Les PED ne seraient pas exemptés de ces normes et standards, mais il leur serait accordé une aide financière pour s'y conformer.

La troisième repose sur un système de taxes nationales harmonisées sur tous les GES dans tous les pays émetteurs, les taxes pouvant être réajustées à intervalles réguliers. Cette approche présuppose la conviction que seul le changement de prix des biens et des services utilisant de l'énergie fossile pourra modifier les décisions de consommations quotidiennes des ménages et des entreprises. Par conséquent, pour restreindre les activités émettrices de $\mathrm{CO}_{2}$, leurs prix doivent augmenter. Chaque pays collecterait ses taxes et gérerait comme il l'entend le revenu obtenu. Le système simple est d'autant plus efficace que le même niveau de taxes négocié internationalement s'impose partout et qu'il est fixé à la source de production ${ }^{49}$. Tous les pays, reconnaît le rapport, devraient avoir les capacités administratives suffisantes pour appliquer de tels plans. Les questions d'équité entre pays ne sont pas ici couvertes par le mécanisme et devraient être négociées à travers des transferts financiers supplémentaires ou d'autres voies d'assistance des pays développés vers les PED.

Enfin, la dernière approche s'appuie sur des politiques nationales allant de pair avec des systèmes de permis d'émissions échangeables (dans des marchés nationaux ou régionaux), liés directement ou indirectement (selon un mécanisme faisant suite au mécanisme de développement propre). En cas d'échec à Copenhague, elle peut apparaître de facto comme le prolongement continu, sans accord général, de la situation actuelle.

Le choix ultime sera celui des politiques. Toutefois, les universitaires de Harvard ne se privent pas de certains jugements : ainsi, le système des taxes, généralement perçu comme le plus efficace et défendu à Poznan par le gouvernement suisse, n'a, selon eux, aucune chance d'être accepté par les pouvoirs politiques (aversion à l'impôt dans le monde) et ils justifient sa mise à l'écart rapide dans le rapport ${ }^{50}$. La seconde approche a l'avantage de ne pas agréger tous les types d'émissions et tous les

\footnotetext{
49 La taxe pour le gaz naturel est fixée aux points de collecte des pipe-lines, celle du pétrole aux raffineries, celle du charbon dans les mines, etc.

${ }^{50}$ Fin 2008, l'économiste américain William Nordhaus - généralement opposé à toute politique climatique ambitieuse s'est prononcé en faveur d'une taxe (très faible) sur le carbone. Le débat a été relancé aux États-Unis à ce sujet (cf. Nordhaus, W., 2008. A Question of Balance: Weighing the Options on Global Warming Policies, New Haven, Yale University Press).
}

secteurs, et d'être plus flexible pour les défis de long terme du changement climatique. Elle est visiblement celle qui leur semble pragmatiquement la plus efficace. D'ailleurs, le WRI, proche lui aussi des milieux démocrates, a fait circuler un rapport approfondi sur l'efficacité et les avantages des approches sectorielles, auxquelles la Chine n'est pas hostile, pour les futurs accords climatiques internationaux ${ }^{51}$.

\section{Équité et développement}

Inclure les PED dans un traité futur sur le climat pose forcément des questions d'équité et de responsabilité. Comment se pose la question de l'équité ou comment définir (et calculer) la «formule» du burden sharing («partage du fardeau») ? Question passionnante et cruciale pour qu'un accord global ait quelques chances d'être accepté, puisqu'un des enjeux est d'associer progressivement les grands pays émergents aux efforts de réduction des émissions. En effet, le Protocole de Kyoto, même s'il était pleinement respecté par les pays signataires, ne couvre $\mathrm{qu}^{\prime}$ une fraction des émissions actuelles et une fraction de plus en plus faible des émissions futures. Des pays à forte croissance économique, comme la Chine, l'Inde et le Brésil, n'ont pas d'objectifs chiffrés. Les inclure dans un futur traité avec des objectifs contraignants - comme l'Europe et les États-Unis le souhaitent - implique de faire des catégories parmi les pays en développement ${ }^{52}$, jusqu'ici unis dans le G77 + Chine, ce qu'ils ne sont pas prêts à accepter.

Associer ces pays aux négociations sur des réductions d'émissions suppose que soit traitée en amont la question $\mathrm{du}$ droit au développement ${ }^{53}$. Or, comment imaginer un développement équitable dans un monde menacé par le changement climatique? Les PED défendent un

\footnotetext{
${ }^{51}$ Cf. Bradley, R., Baumert, K.A., Childs, B., Herzog, T., Pershing, J., 2007. Slicing the Pie: Sector-based Approaches to International Climate Agreements. WRI Report, Washington (http: //pdf.wri.org/slicing-the-pie.pdf). Depuis début 2009, Jonathan Pershing est l'adjoint direct de Todd Stern, en charge du dossier climatique dans l'administration Obama.

${ }^{52}$ Le GIEC propose des « déviations substantielles de la ligne de référence » pour les économies émergentes en 2020 ou, selon l'intensité carbone et la puissance économique des pays, en 2050 (cf. IPCC, 2007. Climate Change 2007: Mitigation. Contribution of Working Group III to the Fourth Assessment Report of the Intergovernmental Panel on Climate Change [Metz, B., Davidson, O.R., Bosch, P.R., Dave, R., Meyer, L.A. (Eds)], Cambridge University Press, Cambridge (UK) and New York, p. 776).

53 Cf. Radanne, P., 2008. La négociation sur le climat à l'ouverture de la Conférence de Poznan, L'Encyclopédie du développement durable, 76 (http://encyclopedie-dd.org/ La-negociation-sur-le-climat-a-1).
} 
«droit égal à l'atmosphère commune ${ }^{54}$ » qui les conduit à l'idée de «convergence par tête à long terme » des émissions de gaz à effet de serre ${ }^{55}$. Ce cadrage de la question de l'équité en termes d'émissions par tête a semblé s'imposer dans le in comme dans le off ${ }^{56}$.

En revanche, la question de la « responsabilité historique », c'est-à-dire le constat que le développement des pays industrialisés est passé par une occupation disproportionnée de l'atmosphère commune, et que les effets déjà observables du changement climatique sont essentiellement le résultat des émissions du Nord, divise fortement. En effet, l'application du principe «pollueur-payeur » dans une perspective historique pose des problèmes d'ordre éthique (une grande partie des émissions date d'une période où l'effet de serre anthropique était mal connu) et scientifique (de nombreuses incertitudes persistent quant à l'attribution par pays du réchauffement anthropique) ${ }^{57}$.

Selon le rapport de Harvard, une voie pragmatique pour la formule du burden sharing serait de commencer par se concentrer sur ce qui est politiquement possible et d'identifier une allocation de responsabilité avec des charges appropriées, de façon que chaque pays ait le sentiment que les exigences à son égard sont équitables.

Sur ces questions éthiques, les arènes climatiques représentent une des premières tentatives de gérer un «bien commun global ». Dans un monde où le développement passerait par une occupation partielle de notre " atmosphère commune » (shared atmosphere), ces négociations doivent à la fois trouver une façon de rendre compte de l'occupation, aujourd'hui très inégale, de cet espace (principe du pollueur-payeur, compensations financières, etc.) et définir un horizon équitable pour le futur. Ces réflexions en sont à leurs débuts, mais les forums climatiques apparaissent comme un champ d'expérimentation pour d'autres défis analogues à venir (biodiversité, eau, etc.) et la structure du processus laisse entrevoir ce «moment

\footnotetext{
54 "China said that the principles of differentiated responsibilities and equity would require allowing developing countries the space to develop. The limited atmospheric resources have been excessively occupied by the developed countries": Lim Li Lin, 2008. Parties reiterate views at Shared Vision contact group, TWN Poznan News Update, 9, 3-4 (http://www.twnside.org.sg/ climate.news.poznan.htm).

${ }_{55}$ Le concept d'une convergence sur le long terme des émissions de gaz à effet de serre par tête a été proposé par Agarwal et Narain dans un livre très controversé (Agarwal, A., Narain, S. 1990. Global Warming in an Unequal World: A Case of Environmental Colonialism, New Delhi, Centre for Science and Environment), avant d'être repris et développé.

${ }^{56}$ La proposition de la Suisse part de ce principe et plusieurs side events sur le futur du processus, dont ceux organisés par les universités Harvard et du Sussex, prennent comme cadre d'analyse la convergence par tête des émissions.

57 Cf. den Elzen, M., Schaeffer, M., 2000. Assessment of Major Uncertainties in Calculating Regional Contributions to Climate Change. RIVM Report 728001012 (http://www.rivm.nl/ bibliotheek/rapporten/728001012.html).
}

cosmopolitique » inhérent, selon Ulrich Beck, aux risques globaux ${ }^{58}$. Au-delà des hésitations, des reculs et des lenteurs, en dépit de larges zones aveugles, les CoP sont inscrites de ce point de vue dans une dynamique qui a fait émerger des questions profondes et stratégiques de notre futur.

\section{Le paradoxe de l'échéance de Copenhague}

Les PED avaient insisté, dès le tout début de la Conférence de Poznan, pour que les discussions sur la « vision commune » ne se concentrent pas seulement sur l'atténuation $^{59}$. Ils faisaient d'un accord sur l'adaptation, le financement et l'échange de technologies un préalable aux négociations sur des objectifs contraignants à moyen et à long terme $\mathrm{e}^{60}$. Ils ont réussi à imposer leurs vues à travers plusieurs points de l'ordre du jour des négociations : mécanisme REDD sur les forêts, financements de $1^{\prime}$ adaptation ${ }^{61}$, «vision commune », bien que, sur ces deux derniers points, les débats se soient achevés à Poznan par un couac.

Du côté de la nouvelle administration américaine, on a vu s'esquisser (à travers le projet de Harvard et d'autres prises de positions ultérieures) l'espace des profils économiques possibles de la négociation : prépondérance toujours aussi forte des mécanismes de marché, apparition de l'idée d'accords sectoriels et relative discrétion sur les formes géopolitiques de gouvernance nécessaires à chacune des options proposées. Cela laisse penser que le rôle de l'ONU n'est plus un objet de litige et que le régime de gouvernance va perdurer peu ou prou à l'identique. En revanche, jusqu' où les États-Unis, l'Europe ou le Japon iront-ils dans leurs objectifs de réductions domestiques et, surtout, dans leurs efforts financiers pour les PED? Quels seront les jeux de pouvoir entre les États-Unis, la Chine et les grands émergents? À l'évidence, rien n'est acquis et l'on perçoit nettement, avec l'approfondissement de la crise économique actuelle, que le régime climatique reste

\footnotetext{
58 Beck, U., 2009. World at Risk, Cambridge (UK), Polity Press (chapitres 3 et 5).

59 Cf. Raman, M., 2008. "Shared vision" debate dominates Poznan's opening plenary, TWN Poznan News Update, 2, 2 (http://www.twnside.org.sg/climate.news.poznan.htm).

60 "Costa Rica said [...] that the shared vision is composed of the four building blocks of the Bali Action Plan [...]. [...] it said that developed country Parties must fully implement their commitments to provide new and additional, adequate and predictable financial resources, necessary for mitigation and adaptation in developing countries": Lim Li Lin, 2008 Parties reiterate views at Shared Vision contact group, TWN Poznan News Update, 9, 1 (http://www.twnside.org.sg/climate. news.poznan.htm).

${ }^{61}$ Cette question mérite d'amples développements, elle est traitée dans le rapport cité en note 1 (voir pp. 32-36).
} 
subordonné à d'autres terrains âpres de compétitions et d'intérêts.

Le paradoxe de l'échéance de Copenhague est qu'un échec serait gravissime (effets démobilisateurs, retards catastrophiques) et qu'un succès relatif ne garantit rien. En effet, face à la lenteur et aux complexités des négociations en cours, face aux nombreuses questions (même légitimes) qui s'y introduisent, force est de constater que l'on perd trop souvent de vue l'objectif ultime de la Convention climat : la réduction des émissions de gaz à effet de serre en vue d'enrayer le changement climatique, une des graves menaces du XXI ${ }^{\mathrm{e}}$ siècle. Il est difficile d'imaginer qu'il sera possible d'atteindre ce but sans sacrifices et sans leadership, sans mesures claires, ambitieuses et compréhensibles en dehors des cercles d'initiés que représentent les CoP. Les discussions autour d'objectifs de réduction des émissions de GES, dans un futur aussi lointain que 2050, ainsi que la préférence pour des solutions technologiques, dont tout reste à inventer, occultent le fait qu'il existe des solutions plutôt simples, tenues pour efficaces - même si elles sont impopulaires -, comme les taxes.

Rappelons que l'Union européenne a essayé à deux reprises de négocier un régime d'écotaxes entre ses Étatsmembres, avant le Sommet de Rio en 1992 et avant la CoP1 de Berlin en $1995^{62}$. Les deux tentatives ont échoué faute de volontarisme politique de certains États ; l'un des résultats de ces échecs est la focalisation actuelle des négociations sur des objectifs à moyen ou long terme et non sur des mesures concrètes. Or, ce mode de gouvernance, qui repose sur des promesses et déplace les arbitrages nécessaires dans le futur ${ }^{63}$, évite les vrais débats, atténue l'importance des changements structurels à venir, ne désigne que des gagnants, jamais de perdants ; il donne finalement trop de marges de manœuvre à toutes sortes de manipulations des «bilans carbone »-du hot air russe aux défaillances des mécanismes de développement propre qui risquent de décrédibiliser le processus sur le moyen terme. Il semble donc urgent de redonner une lisibilité et un vrai enjeu aux négociations, en complétant le débat sur les objectifs par des initiatives sur des mesures concrètes à l'impact immédiat. Et l'Europe, qui s'était voulue la bonne élève du processus mais n'a pas jusqu'ici infléchi de façon décisive le cours des choses, aurait là une initiative de portée historique à prendre.

Les problèmes posés par l'avenir de l'humanité sur notre planète sont désormais liés aux réponses au défi climatique. Celles-ci ne peuvent se réduire ni à une affaire de technologies propres ou vertes, et donc de sciences de l'ingénieur, ni à une affaire d'économistes, bien que ces deux ordres de réponses soient nécessaires. La combinaison d'innovations techniques et d'innovations sociales dans les modes de vie (habitations, territoires) doit absolument s'inscrire dans un projet philosophique d'équité humaine à l'échelle mondialisée, qui fasse droit au caractère multidimensionnel du développement humain. Nous ne disposons pas de critères simples pour l'appréhender. Selon quelles modalités, avec quelles formes politiques de démocratie, à l'échelle mondiale, un nouveau futur peut-il être imaginé et rendu désirable pour le plus grand nombre? Tout reste à inventer, dans et hors les arènes climatiques.

${ }^{62}$ Cf. Hourcade, J.-C., 2001. Le climat au risque de la négociation internationale ?, Le Débat, 113, 137-145.

63 Aykut, S., thèse en cours sur « le global et le local dans les sciences et les politiques du changement climatique ». 